mice showed a significant reduction in the cardiomyocyte cross sectional area (sham, $267 \pm 3.4 \mu \mathrm{m}^{2}$, vehicle treated TAC mice, $480 \pm 5.8 \mu \mathrm{m}^{2}$, AP2 treated TAC mice, $\left.319 \pm 3.9 \mu \mathrm{m}^{2}\right)$. A significant reduction in the expression of the hypertrophic marker ANP and BNP and in the percentage of fibrosis was also observed in these mice compared with vehicle treated TAC mice. AP2 treatment led to a significant reduction in the expression of the bona fide calcineurin target RCAN1.4 and a reduction in the NFAT phosphorylation level in vivo and the NFAT transcriptional activity in vitro. In conclusion, we have identified AP2 as a novel PMCA4 specific inhibitor and shown its potential to modify the development of cardiac hypertrophy likely through inhibition of calcineurin/NFAT signalling. This compound has drug-like properties and thus lays the basis for a novel approach for treating cardiac hypertrophy and failure through PMCA4 inhibition.

\section{CHARACTERISATION OF FRACTIONATED ATRIAL ELECTROGRAMS CRITICAL FOR MAINTENANCE OF AF: A RANDOMISED CONTROLLED TRIAL OF ABLATION STRATEGIES (THE CFAE AF TRIAL)}

doi:10.1136/heartjnl-2011-300198.145

R J Hunter, I Diab, M Tayebjee, L Richmond, S Sporton, M J Earley, R J Schilling. Barts and The London NHS Trust, London

Introduction Targeting complex fractionated atrial electrograms (CFAE) in the ablation of atrial fibrillation (AF) may improve outcomes, although whether this is by eliminating focal drivers or simply de-bulking atrial tissue is unclear. It is also uncertain what electrogram morphology should be ablated. This randomised study aimed to determine the impact of ablating different CFAE morphologies compared to normal electrograms (ie, de-bulking normal tissue) on the cycle length of persistent AF (AFCL).

Methods After pulmonary vein isolation CFAE were targeted systematically throughout the left then right atrium, until termination of AF or abolition of CFAE prior to DC cardioversion. $10 \mathrm{~s}$ electrograms were classified by visual inspection according to a validated scale, with Grade 1 being most fractionated and grade 5 normal. Patients were randomised to have CFAE grades eliminated sequentially, from grade 1 to 5 (group 1) or grade 5 to 1 (group 2). Because grade 5 electrograms were considered normal, only 5 were ablated. Mean AFCL was determined manually over 30 cycles from bipolar electrograms recorded at the left and right atrial appendages before and after each CFAE was targeted. Lesions were regarded as individual observations, and a resultant increase in mean AFCL $\geq 5 \mathrm{~ms}$ was regarded as significant. The randomised strategy first controlled for any cumulative effect of ablation on AFCL, and second allowed assessment of the order of ablation on the number of CFAE lesions required.

Results 20 patients were randomised. The CFAE grade determined by rapid visual inspection for the 968 electrograms targeted agreed with that at off-line manual measurement in $92.7 \%(\mathrm{~K}=0.91)$. AFCL increased after targeting $49.5 \%$ of grade 1 CFAE, $33.6 \%$ of grade 2 , $12.8 \%$ of grade $3,33.0 \%$ of grade 4 , and $8.2 \%$ of grade 5 CFAE ( $p<0.0001$ for grades 1,2 , and 4 vs 5,3 vs 5 not significant). Binary logistic regression confirmed the effect of CFAE grade, but showed no effect of electrogram amplitude, location in the left or right atrium, or the order in which CFAE were targeted. There was no difference between groups in the number of grade 1 or 2 CFAE encountered, but there were fewer grade 3 and 4 CFAE in group 2 than group 1 (both $\mathrm{p}<0.01)$, translating to fewer CFAE targeted per patient in group 1 compared to group 2 ( $37 \pm 14$ and $58 \pm 18$ respectively; $\mathrm{p}=0.015)$.

Conclusion Targeting CFAE is not simply atrial de-bulking. Ablating certain grades of CFAE caused AFCL prolongation, suggesting they are more important in maintaining AF. Targeting these CFAE may reduce unnecessary left atrial destruction. (ClinicalTrials.gov number, NCT00894400).

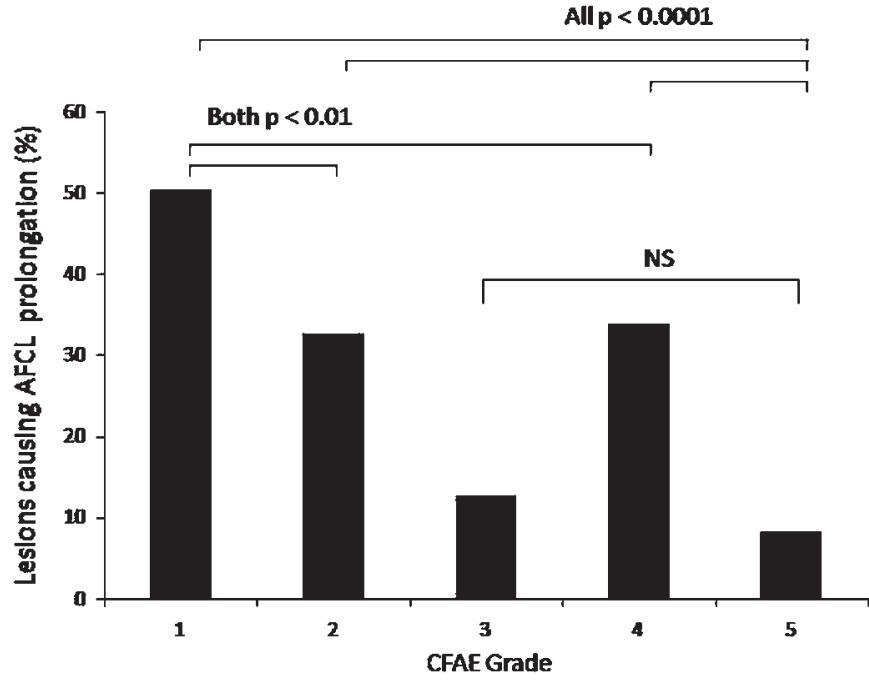

Abstract 145 Figure 1 Impact of CFAE grade on the proportion of lesions causing AF cycle length prolongation.

\section{IS THERE AN ASSOCIATION BETWEEN THROMBOGENESIS MARKERS AND ATRIAL FIBRILLATION BURDEN IN PACEMAKER POPULATION?}

doi:10.1136/heartjnl-2011-300198.146

C W Khoo, S Krishnamoorthy, G Dwivedi, B Balakrishnan, HS Lim, G Y H Lip. University Department of Medicine Centre for Cardiovascular Sciences, City Hospital, Birmingham, UK

Background and Objectives Contemporary pacemaker devices are able to quantify atrial high-rate episodes (AHREs) and atrial fibrillation burden (AFB) accurately. In this study, we aim to assess the relationship of thrombogenesis markers in association with AHREs and AFB.

Methods We studied 87 patients with dual-chamber pacemaker. Patients on warfarin were excluded. AHREs were defined as atrialrate $\geq 220$ beats $/ \mathrm{min}$ and $\geq 5$ minutes. AFB and percentage of cumulative pacing were derived from pacemaker diagnostics. Plasma levels of von Willebrand factor (vWf), tissue factor (TF), soluble P-selectin (P-sel) and D-dimer (DDM) were analysed using ELISA.

Results Baseline characteristics and co-morbidities were comparable between groups (Abstract 146 table 1). Patients with AHREs had significantly higher cumulative percentage ventricular pacing $(p=0.012)$. There were no significant differences in levels of vWf, TF, P-sel and DDM between patients with and without AHREs. The AFB ranged from 0 to $99 \%$ in AHRE group. TF ( $r=0.516, p=0.086)$, P-sel $(r=0.795, p<0.001)$ and DDM $(r=0.643, p=0.045)$ correlated with AFB. On linear regression analysis, both P-sel and DDM were independently associated with AFB $(p<0.05)$.

\section{Abstract 146 Table 1}

\begin{tabular}{llll}
\hline & No AHRE $(\mathbf{n}=70)$ & AHRE $(\mathbf{n}=17)$ & p value \\
\hline Age, years & $71.0 \pm 11.6$ & $75.4 \pm 8.8$ & 0.096 \\
Hypertension, (\%) & $38,(54)$ & $12,(71)$ & 0.116 \\
Antiplatelet, (\%) & $53,(76)$ & $14,(82)$ & 0.739 \\
Percentage atrial pacing & $34.6(6.8-81.5)$ & $22.1(6.9-65.0)$ & 0.414 \\
Percentage ventricular pacing & $21.9(1.8-99.0)$ & $98.6(41.0-99.9)$ & 0.012 \\
vWf, IU/dl & $94.2 \pm 16.2$ & $93.9 \pm 33.7$ & 0.977 \\
TF, ng/ml & $0.2(0.1-0.3)$ & $0.1(0.0-0.2)$ & 0.105 \\
P-sel, ng/ml & $47.6 \pm 15.8$ & $63.4 \pm 29.7$ & 0.055 \\
$\mathrm{DDM}, \mathrm{ng} / \mathrm{ml}$ & $180.0(82.0-390.0)$ & $152.5(82.5-307.5)$ & 0.553 \\
\hline
\end{tabular}


Conclusion AFB is independently associated with increased indices of P-sel and D-dimer which indicate platelet activation and thrombosis respectively.

\section{THROMBOEMBOLIC RISK STRATIFICATION, ANTI- THROMBOTIC AND ANTICOAGULATION USE FOR PATIENTS WITH ATRIAL FIBRILLATION, A CLINICAL AUDIT}

doi:10.1136/heartjnl-2011-300198.147

R A Veasey, R Kulanthaivelu, P Patel, D W Harrington. Kent and Sussex Hospital, Tunbridge Wells, UK

Introduction Atrial fibrillation (AF) is the most prevalent arrhythmia in primary and secondary healthcare settings. Thromboembolic (TE) risk assessment and initiation of anti-thrombotic or anticoagulation (AT/AC) therapy, according to level of risk, is recommended in both national and international guidelines. NICE guidance stratifies patients with AF in to low, moderate or high risk categories and recommends "aspirin", "aspirin or warfarin" or "warfarin" therapy respectively. ACC/ESC guidance endorses use of the $\mathrm{CHADS}_{2}$ scoring system and for scores of 0,1 , or $\geq 2$ recommends "aspirin", "aspirin or warfarin" or "warfarin" therapy respectively. In addition, it is recommended that AF episode frequency or subtype (paroxysmal (PAF), persistent (PersAF) or chronic (CAF)) does not influence TE risk assessment. We audited UK cardiologists and general practitioners (GPs) to assess adherence to these guidelines.

Methods We designed an audit questionnaire assessing: (1) use of risk stratification tools, (2) choice of AT/AC for increasing levels of risk, and (3) choice of therapy for a number of hypothetical patients with variable TE risk and variable AF subtype. The questionnaire was distributed by electronic or postal mail to 1176 cardiologists and 621 randomly selected GPs.

Results In total, 421 responses were received (306 cardiologists, 115 GPs). Overall, $91.4 \%$ of responders reported use of TE risk stratification tools (97.1\% cardiologists, $76.5 \%$ GPs, $\mathrm{p}<0.001)$. NICE risk assessment is used by $26.6 \%$ of responders $(24.5 \%$ cardiologists, $32.2 \%$ GPs, $\mathrm{p}=0.14$ ), $\mathrm{CHADS}_{2}$ by $79.3 \%$ (90.2\% cardiologists, $50.0 \%$ GPs, $p<0.001)$. The frequency of reported use of AT/AC for each risk level of the NICE assessment and $\mathrm{CHADS}_{2}$ score are shown in Abstract 147 tables 1 and 2 respectively. Type of AF (PAF/PersAF/ $\mathrm{CAF}$ ) reportedly influences the use of $\mathrm{AT} / \mathrm{AC}$ for $34.3 \%$ or responders (24.2\% cardiologists, 46.3\% GPs, $\mathrm{p}=0.001)$. Abstract 147 figure 1 demonstrates AT/AC usage for each of the following hypothetical patients: 1 . 61 year old, hypertension, PAF episodes twice a year lasting 1-2 h (NICE risk: $\bmod , \mathrm{CHADS}_{2}$ score 1/6). 2. 43 year old, diabetes, PAF episodes weekly lasting 10-12 h (NICE risk: mod, $\mathrm{CHADS}_{2}$ score 1/6). 3. 53 year old, hypertension, CAF (NICE risk: mod, $\mathrm{CHADS}_{2}$ score 1/6). 4.78 year old, no other risk factors, CAF (NICE risk: mod, $\mathrm{CHADS}_{2}$ score 1/6). 5. 76 year old, hypertension, diabetes, PAF episodes 3-4 times per year lasting $<1$ hour (NICE risk: high, $\mathrm{CHADS}_{2}$ score 3/6). 6. 77 year old, hypertension, diabetes, PAF episodes occurring weekly and lasting several hours (NICE risk: high, $\mathrm{CHADS}_{2}$ score 3/6). 7. 80 year old, previous TIA, CAF (NICE risk: high, $\mathrm{CHADS}_{2}$ score 3/6).

Abstract 147 Table 1

\begin{tabular}{lcccc}
\hline NICE Risk & None (\%) & Aspirin (\%) & Aspirin or Warfarin (\%) & Warfarin (\%) \\
\hline Low & 16.7 & 78.3 & 2.9 & 2.1 \\
Moderate & 0.6 & 3.5 & 66.9 & 28.7 \\
High & 0.0 & 0.0 & 4.4 & 95.6 \\
\hline
\end{tabular}

Abstract 147 Table 2

\begin{tabular}{lcccc}
\hline CHADS2 Score & None $(\%)$ & Aspirin (\%) & Aspirin or Warfarin (\%) & Warfarin (\%) \\
\hline Zero & 27.0 & 70.3 & 1.3 & 1.3 \\
One & 4.7 & 45.1 & 43.2 & 7.0 \\
Two & 0.0 & 7.8 & 32.2 & 60.0 \\
Three & 0.0 & 0.8 & 11.5 & 87.7 \\
Four & 0.0 & 0.0 & 5.3 & 94.7 \\
Five & 0.0 & 0.0 & 2.9 & 97.1 \\
Six & 0.0 & 0.0 & 2.9 & 97.1 \\
\hline
\end{tabular}

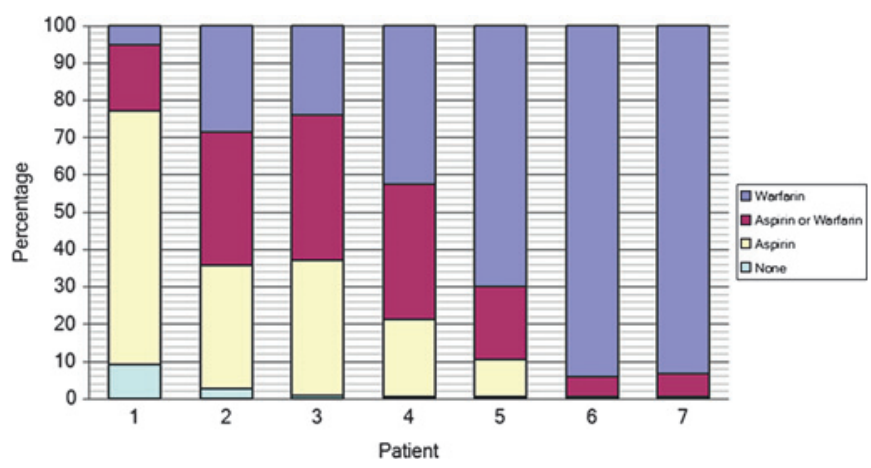

Abstract 147 Figure 1

Conclusions TE risk stratification tools are reportedly widely used in UK clinical practice. AT/AC use for NICE and $\mathrm{CHADS}_{2}$ risk levels are mostly appropriate, although warfarin is under recommended for patients with a $\mathrm{CHADS}_{2}$ score of $2 / 6$. In addition, the use of AT/ $\mathrm{AC}$ is influenced, inappropriately, by AF episode frequency and subtype.

\section{THE ASSESSMENT OF TRANSIENT LOSS OF CONSCIOUSNESS: WE'RE STILL NOT ASKING THE RIGHT QUESTIONS}

doi:10.1136/heartjnl-2011-300198.148

A E Bewick, A Gasson, L Ala, R A Bleasdale. Royal Glamorgan Hospital, Cardiff, UK

Accurately diagnosing patients with TLOC can be achieved in most cases with a detailed clinical history. We set out to assess how patients were assessed in the setting of a district general hospital (DGH) with 570 beds, receiving an unselected intake via general practice and an A\&E. Using the ESC guidelines of 2009 we generated a 22-question study proforma for a retrospective review of the medical records. We identified 322 cases for possible inclusion over a 4 month period. 26 of the case notes were not available to analyse, 8 had insufficient details to identify the relevant patient. Therefore in total 288 notes were reviewed. Inclusion required the TLOC to be complete, of rapid onset and short duration with spontaneous complete recovery. A further 123 patients were therefore excluded. This left 165 data sets (58\% male). The age distribution was a typical bimodal distribution with $16 \%$ between 10 and 29 years of age and $48 \%$ over the age of 70 years. $73 \%$ were assessed in A\&E, $18 \%$ were assessed in the Acute Medical Unit (AMU) and $7 \%$ were assessed in rapid access ambulatory clinics. Only $4 \%$ of the initial assessments were undertaken by consultants, $12 \%$ by a Specialist Registrar (SpR), 21\% by a year 1 foundation program (FP1) doctor and the majority was assessed by FP 2 or core medical trainees (CMT). Key diagnostic elements of the history are still being neglected. For example, the symptoms at the onset of the TLOC were documented in only $58 \%$ of cases; the recovery symptom 